\title{
Hecke algebraic properties of dynamical $R$-matrices. Application to related quantum matrix algebras
}

\author{
L.K. Hadjiivanov ${ }^{a}$ 中, A.P. $\operatorname{Isaev}^{b}$ ใ, O.V. Ogievetsky ${ }^{c}$ ? \\ P.N. Pyatov ${ }^{d}$ 由 and I.T. Todorov ${ }^{e}$ 向 \\ ${ }^{a}$ International Centre for Theoretical Physics (ICTP), I-34014 Trieste, Italy \\ ${ }^{b}$ Dipartimento di Fisica, Università di Pisa, I-56100 Pisa, Italy \\ ${ }^{c}$ Centre de Physique Théorique, Luminy, F-13288 Marseille, France \\ ${ }^{d}$ Bogoliubov Laboratory of Theoretical Physics, JINR, \\ Dubna, 141980 Moscow Region, Russia \\ ${ }^{e}$ Erwin Schrödinger Institute for Mathematical Physics (ESI), \\ A-1090 Wien, Austria
}

\begin{abstract}
The quantum dynamical Yang-Baxter (or Gervais-Neveu-Felder) equation defines an $R$-matrix $\hat{R}(p)$, where $p$ stands for a set of mutually commuting variables. A family of $S L(n)$-type solutions of this equation provides a new realization of the Hecke algebra. We define quantum antisymmetrizers, introduce the notion of quantum determinant and compute the inverse quantum matrix for matrix algebras of the type $\hat{R}(p) a_{1} a_{2}=a_{1} a_{2} \hat{R}$. It is pointed out that such a quantum matrix algebra arises in the operator realization of the chiral zero modes of the WZNW model.
\end{abstract}

\footnotetext{
${ }^{1}$ On leave of absence from: Division of Theoretical Physics, Institute for Nuclear Research and Nuclear Energy, Bulgarian Academy of Sciences, Tsarigradsko Chaussee 72, BG-1784 Sofia, Bulgaria; e-mail address: lhadji@inrne.acad.bg

${ }^{2}$ On leave of absence from: Bogoliubov Laboratory of Theoretical Physics, JINR, Dubna, 141980 Moscow Region, Russia; e-mail address: isaevap@thsun1.jinr.ru

${ }^{3}$ On leave of absence from: P.N. Lebedev Physical Institute, Theoretical Department, 117924 Moscow, Leninsky prospect 53, Russia; e-mail address: oleg@cpt.univ-mrs.fr

${ }^{4}$ e-mail address: pyatov@thsun1.jinr.ru

${ }^{5}$ On leave of absence from: Division of Theoretical Physics, Institute for Nuclear Research and Nuclear Energy, Bulgarian Academy of Sciences, Tsarigradsko Chaussee 72, BG-1784 Sofia, Bulgaria; e-mail address: todorov@inrne.acad.bg
} 


\section{Introduction}

Let $\left\{v^{(i)}, i=1, \ldots, n\right\}$ be a "barycentric basis" in a Cartan subalgebra $\mathfrak{h} \subset$ $s l(n)$. Viewed as operators in the $n$-dimensional complex space $V=\mathbb{C}^{n}, v^{(i)}$ can be realized as real traceless diagonal $n \times n$ matrices:

$$
\left(v^{(i)}\right)_{j}^{j}=\delta_{i j}-\frac{1}{n}, \quad \sum_{i=1}^{n} v^{(i)}=0 .
$$

Let further $\left\{p_{i}\right\}_{i=1}^{n}$ span the dual Lie algebra $\mathfrak{h}^{*}$. Introduce the traceless diagonal matrix

$$
p=p_{i} v^{(i)}\left(\equiv \sum_{i=1}^{n} p_{i} v^{(i)}\right), \quad\left[p_{i}, p_{j}\right]=0, \quad \sum_{i=1}^{n} p_{i}=0 .
$$

We define a Hecke-type quantum dynamical $R$-matrix $\hat{R}(p)$ as a map from $\mathfrak{h}^{*}$ to End $(V \otimes V)$ satisfying the twisted braid relation

$$
\hat{R}_{12}(p) \hat{R}_{23}\left(p-v_{1}\right) \hat{R}_{12}(p)=\hat{R}_{23}\left(p-v_{1}\right) \hat{R}_{12}(p) \hat{R}_{23}\left(p-v_{1}\right)
$$

and the Hecke condition

$$
\hat{R}(p)^{2}=\mathbb{I}+(q-\bar{q}) \hat{R}(p), \quad \bar{q}:=q^{-1} .
$$

(Although the notation is taylored to the special case in which the parameter $q$ takes values on the unit circle, we shall not use this property in the main body of the paper.) The subscripts in (0.3) refer to the, by now standard, tensor product notation of Faddeev et al. (see, e.g., [1]); in particular, $\hat{R}_{23}(p-$ $\left.v_{1}\right) \in \operatorname{End}\left(V^{\otimes 3}\right)$ has matrix elements

$$
\left(\hat{R}_{23}\left(p-v_{1}\right)\right)_{j_{1} j_{2} j_{3}}^{i_{1} i_{2} i_{3}}=\delta_{j_{1}}^{i_{1}} \hat{R}\left(p-v^{\left(i_{1}\right)}\right)_{j_{2} j_{3}}^{i_{2} i_{3}} .
$$

The twisted braid relation (0.3) is equivalent to the quantum dynamical [2, [3] (or deformed [4]) Yang-Baxter equation (QDYBE) for the matrix $R(p)$ related to the braid operator $\hat{R}(p)$ by $\hat{R}(p)=P R(p)$ where $P$ stands for the permutation operator $P x_{1} y_{2}=y_{1} x_{2}, x, y \in V, P^{2}=1$. Abusing notation we shall also refer to Eq.(0.3) by the above abbreviation. The term "dynamical $R$-matrix" for $\hat{R}(p)$ is suggested by the fact that in the physical applications 
its arguments play the role of (commuting) dynamical variables and that $\hat{R}(p)$ satisfies a finite difference rather than a purely algebraic equation.

The important concept of a quantum matrix algebra $\mathcal{A}=\mathcal{A}(\hat{R}(p), \hat{R})$ (Sec.5) can be introduced as a (complex) associative algebra with 1 generated by rational functions of $q^{p_{i}}, i=1, \ldots, n$, and the (noncommuting) entries of an $n \times n$ matrix $a=\left(a_{\alpha}^{i}\right)$ satisfying the quadratic exchange relations

$$
\hat{R}(p) a_{1} a_{2}=a_{1} a_{2} \hat{R}
$$

where $\hat{R} \equiv \hat{R}_{\beta_{1} \beta_{2}}^{\alpha_{1} \alpha_{2}}$ in the right hand side is a constant (i.e., $p$-independent) solution of (0.3), (0.4), and all entries in a matrix row $a^{i}=\left\{a_{\alpha}^{i}\right\}_{\alpha=1}^{n}$ are acting equivalently as shift operators for $p$ :

$$
p a^{i}=a^{i}\left(p+v^{(i)}\right) \text { or } p_{j k} a^{i}=a^{i}\left(p_{j k}+\delta_{j}^{i}-\delta_{k}^{i}\right) \text { for } p_{j k}=p_{j}-p_{k} .
$$

Remark 1 It has been pointed out [5, [1] that, in the $s u(2)$ case, the matrix a generates the $q$-Clebsh-Gordan coefficients while $\hat{R}(p)$ plays the role of a "quantum 6j-symbol" [6, 7, 8].

Remark 2 Eq.(0.6) is related to the one with indices 1 and 2 interchanged,

$$
\hat{R}(p) a_{2} a_{1}=a_{2} a_{1} \hat{R}
$$

by the substitution $\hat{R} \rightarrow P \hat{R} P, \hat{R}(p) \rightarrow P \hat{R}(p) P$. It is, on the other hand, formally obtained from

$$
\hat{R} \bar{a}_{2} \bar{a}_{1}=\bar{a}_{2} \bar{a}_{1} \hat{R}(p) .
$$

by the substitution $\bar{a}=a^{-1}$; the same substitution relates (0.8) to

$$
\hat{R} \bar{a}_{1} \bar{a}_{2}=\bar{a}_{1} \bar{a}_{2} \hat{R}(p) .
$$

Since

$$
\hat{R}(p)_{21}=P \hat{R}(p) P
$$

satisfies conditions of the same type as $\hat{R}(p)$, we can start with either of these relations.

The QDYBE, introduced by Gervais and Neveu [2] for the exchange algebra associated with the Liouville equation and applied to the zero mode algebra [5] of the Wess-Zumino-Novikov-Witten (WZNW) model [9, 10, 11] is attracting ever more attention. Its classical counterpart, introduced in [3] (see also [12]) has been displayed in [5] for the $\operatorname{sl}(2)$ case and in [13] for an arbitrary simple Lie algebra. The quantum $\hat{R}(p)$ is central to a continuing study [4] of $q$-deformed cotangent bundles on group manifolds and quantum model spaces. A quasiHopf-algebraic point of view is taken in [14, 15] where $\hat{R}(p)$ is obtained by a Drinfeld twist of the constant $R$-matrix. Felder [16 explores the more general case of (classical and) QDYBE depending on a spectral parameter and finds elliptic solutions of this equation. These 
solutions are applied in [17 to quantize Calogero-Moser and RuijsenaarsSchneider models. A class of $S L(n \mid m)$-type solutions of the QDYBE (and related trigonometric solutions of the equation with spectral parameter) are described in [18]. A more mathematically minded approach to the subject in terms of "h-algebroids" is being developed in 19.

The present work was motivated in part by earlier study [20, 21, 22] of the canonical quantization of the WZNW model (following 16, 10, 5, 11, 231). It was noticed, in particular, that the exchange relations 21] for the chiral zero modes $a_{\alpha}^{i}$ that diagonalize the $U_{q}(s l(2))$ monodromy matrix can be written in the form (0.8). As a result, the operator realization of the chiral group valued field was understood as a quantization of the (deformed) classical Poisson bracket relations of 13 thus opening the way to its generalization for $S U(n)$. Here we show that a special solution of the QDYBE (0.3) yields a new matrix representation of the Hecke algebra. We concentrate on a general study of the Hecke algebra properties of this solution and the ensuing properties of the quantum matrices satisfying (0.6) relegating applications to the WZNW model to a subsequent publication [24] which is highlighted in Section 6. A central result is the computation of the quantum determinant of $a$ and the (based on it) evaluation of the inverse quantum matrix.

The paper is organized as follows. We review and extend in Section 1 results of Gurevich 25] on quantum (anti)symmetrizers and illustrate them in Section 2 on the known example of a constant $\hat{R}$. We proceed in Section 3 to a study of a family of $S L(n)$-type dynamical $R$-matrices and describe two types of symmetry transformations for this family: the twist transformation (a version of Drinfeld's twist for dynamical $R$-matrices) and the canonical shifts. In Section 4 we show that these dynamical matrices provide a new realization of the Hecke algebra. This allows to define "dynamical" ( $p$-dependent) analogs of quantum antisymmetrizers, including the Levi-Cività $\mathcal{E}$ tensor. In Section 5 we study the quantum algebra $\mathcal{A}$, define the quantum determinant $\operatorname{det}(a)$ and compute the inverse matrix $a^{-1}$. We demonstrate that $\mathcal{A}$ provides a realization of a reflection equation algebra which is interpreted as a quantum monodromy algebra in the WZNW theory. An Appendix is devoted to deriving some useful identities for the parameters determining the solution of the QDYBE found in [18] and to computing the normalization of the dynamical Levi-Cività tensor. 


\section{Hecke algebras and $q$-antisymmetrizers}

In this Section we collect some basic notions on Hecke algebras and describe the $q$-antisymmetrizers technique which is to be applied later on. We follow closely the approach of D. Gurevich [25].

In the present context by a Hecke algebra $\mathcal{H}_{k}(q)$ we understand a $\mathbb{C}$ algebra with generators $1, g_{1}, g_{2}, \ldots, g_{k-1}$, a nonzero parameter $q \in \mathbb{C}$, and defining relations

$$
\begin{aligned}
g_{i} g_{i+1} g_{i}=g_{i+1} g_{i} g_{i+1} & \text { for } 1 \leq i \leq k-2, \\
g_{i}{ }^{2}=1+(q-\bar{q}) g_{i} & \text { for } 1 \leq i \leq k-1, \\
g_{i} g_{j}=g_{j} g_{i} & \text { if }|i-j| \geq 2,
\end{aligned}
$$

where $\bar{q}:=q^{-1}$.

We shall consider the set of idempotents $A^{(j)} \in \mathcal{H}_{k}(q), j=1, \ldots, k$, associated with single column Young diagrams containing $j$ nodes - the so called $q$-antisymmetrizers. Their inductive definition is given by

$$
A^{(1)}=1, \quad A^{(j)}=\frac{1}{[j]} A^{(j-1)}\left(q^{j-1}-[j-1] g_{j-1}\right) A^{(j-1)} .
$$

Here $[j]=\left(q^{j}-\bar{q}^{j}\right) /(q-\bar{q})$ and we assume $[j] \neq 0$, for $j=2, \ldots, k$. Note, that $A^{(k)}$ is a central idempotent in the algebra $\mathcal{H}_{k}(q)$.

Equivalently, one may write

$$
A^{(j)}=\frac{1}{[j]} A^{(2, j-1)}\left(q^{j-1}-[j-1] g_{1}\right) A^{(2, j-1)},
$$

where we have adopted the notation $A^{(i, j)}, 1 \leq i \leq j$, for the central idempotent of the subalgebra $\mathcal{H}_{i, j}(q) \subset \mathcal{H}_{j}(q)$ generated by the subset $1, g_{i}, \ldots, g_{j-1}$. In particular, $A^{(1, j)}=A^{(j)}, A^{(j, j)}=1$.

Remark 1.1 All the subalgebras $\mathcal{H}_{i, r+i}(q) \subset \mathcal{H}_{k}(q), i=1, \ldots, k-r$, are isomorphic by definition. Moreover, they are related by inner $\mathcal{H}_{k}(q)$-automorphisms. For example, the automorphism $\phi_{i}: \mathcal{H}_{i, r+i}(q) \rightarrow \mathcal{H}_{i+1, r+i+1}(q)$ is given by

$$
\phi_{i}(t)=g_{i} g_{i+1} \ldots g_{r+i} t\left(g_{i} g_{i+1} \ldots g_{r+i}\right)^{-1}, \quad \forall t \in \mathcal{H}_{i, r+i}(q) .
$$

The term $q$-antisymmetrizer for the elements $A^{(j)}$ is justified by the following properties:

$$
\begin{aligned}
& \left(g_{i}+\bar{q}\right) A^{(j)}=A^{(j)}\left(g_{i}+\bar{q}\right)=0 \quad \text { for } 1 \leq i \leq j-1, \\
& A^{(j)} A^{(i, l)}=A^{(i, l)} A^{(j)}=A^{(j)} \quad \text { for } 1 \leq i \leq l \leq j .
\end{aligned}
$$

Remark 1.2 Replacing $q$ by $\left(-q^{-1}\right)$ in (1.4) leads to another sequence of projectors, called symmetrizers. Abstractly, inside the Hecke algebra, it is a matter of convention - which projectors one calls symmetrizers, and which - 
antisymmetrizers. We use the common convention. However, on the level of representations, when one can calculate the ranks of projectors and see which sequence of projectors terminates, the distinction between symmetrizers and antisymmetrizers becomes meaningful.

Consider a representation $\rho_{W, k}: \mathcal{H}_{k}(q) \rightarrow \operatorname{Aut}(W)$ of the algebra $\mathcal{H}_{k}(q)$ in a vector space $W$.

Definition 1.1 We shall say that $\rho_{W, k}$ is a representation of height $n$ in one of the following two cases:

a) $n<k$ and the conditions

$$
\begin{aligned}
\rho_{W, k}\left(A^{(n+1)}\right) & =0, \\
\operatorname{rank} \rho_{W, k}\left(A^{(n)}\right) & =1,
\end{aligned}
$$

are fulfilled, or

b) $n=k$ and $\quad \operatorname{rank} \rho_{W, n}\left(A^{(n)}\right)=1$.

Remark 1.3 The notion of height of a Hecke algebra representation was introduced in 25 for the special case of the representations generated by constant $R$-matrices. There it was named the rank of the $R$-matrix. We have changed the name here in order to avoid a possible confusion with the standard notion of rank of a matrix. Note that the use of the term 'height' is suggested by the fact that imposing condition (1.9) for the representation $\rho_{W, k}$ results in vanishing of any central (and primitive) idempotent related to a Young diagram (standard tableaux) containing more then $n$ boxes in one of its columns.

Remark 1.4 In view of Remark 1.1, the whole sets of $q$-antisymmetrizers $\left\{\rho_{W, k}\left(A^{(i, n+i)}\right)\right\}_{i=1, \ldots, k-n}$ and $\left\{\rho_{W, k}\left(A^{(j, n+j-1)}\right)\right\}_{j=1, \ldots, k-n+1}$ satisfy conditions (1.9) and (1.10), respectively.

Remark 1.5 Instead of using (1.9) one can impose the condition

$$
A^{(n+1)}=0
$$

at the algebraic level. This is the way how generalized Temperley-LiebMartin algebras are defined (cf. [26]). Below we present several useful equivalent forms of this condition.

Lemma 1.1 The condition (1.11) is equivalent to any of the following relations

$$
\begin{gathered}
A^{(n)} g_{n} \ldots g_{2} g_{1}=(-1)^{n-1} q[n] A^{(n)} A^{(2, n+1)}, \\
g_{1} g_{2} \ldots g_{n} A^{(n)}=(-1)^{n-1} q[n] A^{(2, n+1)} A^{(n)}, \\
g_{n} \ldots g_{2} g_{1} A^{(2, n+1)}=(-1)^{n-1} q[n] A^{(n)} A^{(2, n+1)}, \\
A^{(2, n+1)} g_{1} g_{2} \ldots g_{n}=(-1)^{n-1} q[n] A^{(2, n+1)} A^{(n)}, \\
A^{(n)} A^{(2, n+1)} A^{(n)}=[n]^{-2} A^{(n)}, \\
A^{(2, n+1)} A^{(n)} A^{(2, n+1)}=[n]^{-2} A^{(2, n+1)} .
\end{gathered}
$$


Proof. Applying repeatedly (1.4) for the $q$-antisymmetrizers that appear as last factors in the resulting products and using (1.7), (1.8) we find

$$
\begin{aligned}
A^{(n+1)} & =\frac{1}{[n+1]} A^{(n)}\left(q^{n}-[n] g_{n}\right) A^{(n)} \\
& =\frac{1}{[n+1]}\left\{q^{n} A^{(n)}-A^{(n)} g_{n}\left(q^{n-1}-[n-1] g_{n-1}\right) A^{(n-1)}\right\} \\
& =\frac{1}{[n+1]}\left\{A^{(n)}\left(q^{n}-q^{n-1} g_{n}\right)+A^{(n)} g_{n} g_{n-1}\left(q^{n-2}-[n-2] g_{n-2}\right) A^{(n-2)}\right\} \\
\ldots & =\frac{1}{[n+1]} A^{(n)}\left(q^{n}-q^{n-1} g_{n}+\ldots+(-1)^{n} g_{n} g_{n-1} \ldots g_{1}\right) .
\end{aligned}
$$

Next, we apply $A^{(2, n+1)}$ to the both sides of Eq.(1.13). Using again (1.7) and (1.8) we obtain

$$
A^{(n+1)}=\frac{1}{[n+1]} A^{(n)}\left\{q[n] A^{(2, n+1)}+(-1)^{n} g_{n} g_{n-1} \ldots g_{1} A^{(2, n+1)}\right\} .
$$

Taking into account the relation $\left(g_{n} g_{n-1} \ldots g_{1}\right) A^{(2, n+1)}=A^{(n)}\left(g_{n} g_{n-1} \ldots g_{1}\right)$ which is a consequence of (1.1) we end up with

$$
A^{(n+1)}=\frac{1}{[n+1]}\left\{q[n] A^{(n)} A^{(2, n+1)}+(-1)^{n} A^{(n)} g_{n} g_{n-1} \ldots g_{1}\right\} \text {. }
$$

This proves the equivalence of Eqs.(1.11) and (1.12a). A similar argument using iteratively a substitution of the first $q$-antisymmetrizer in the right hand side of (1.4) implies the equivalence of (1.11) and (1.12b). Condition (1.11) is transformed to the forms $(1.12 \mathrm{c})$ and $(1.12 \mathrm{~d})$ in the same manner starting from Eq.(1.5).

To show equivalence of (1.11) to (1.12e) and to (1.12f) one should employ Eqs.(1.4) and(1.5), respectively. We shall treat the case of (1.12e) here.

Consider the difference

$$
\begin{aligned}
& {[n]^{2} A^{(n)} A^{(2, n+1)} A^{(n)}-A^{(n)}=A^{(n)}\left([n]^{2} A^{(2, n+1)}-1\right) A^{(n)} } \\
= & A^{(n)}\left\{[n] A^{(2, n)}\left(q^{n-1}-[n-1] g_{n}\right) A^{(2, n)}-1\right\} A^{(n)} \\
= & {[n-1] A^{(n)}\left(q^{n}-g_{n}\right) A^{(n)}=[n-1][n+1] A^{(n+1)}, }
\end{aligned}
$$

where we have again used the definition (1.4) and the relations (1.8). Comparing the first and the last lines of the calculation we deduce the equivalence of conditions (1.11) and (1.12e).

Eqs.(1.12a-f) display properties of the rank 1 idempotents $\rho_{W, k}\left(A^{(n)}\right)$ that are hidden in (1.11). In fact, they are the basic technical tools which one needs to effectively deal with the height $n$ Hecke algebra representations.

In the rest of the paper we make use of a special type of representations of the algebras $\mathcal{H}_{k}(q)$ for which the representation space is given by $k$-th tensor power of an ( $n$-dimensional, in the case of $S L(n))$ vector space $V$ : $W=V^{\otimes k}$. These representations are generated by constant or dynamical 
$R$-matrices of Hecke type. The representations we are dealing with have the specific feature that their height, when defined (i.e., for $k \geq n$ ), coincides with the dimension of the space $V$ 円. Below we first illustrate the general notions introduced above on the well known case of constant $S L(n)$-type $R$-matrices relegating the study of dynamical $R$-matrices to Sections 3 and 4 .

\section{Representations generated by a constant $R$-matrix of $S L(n)$ type}

The $R$-matrix corresponding to the Drinfeld-Jimbo deformation of $S L(n)$ 27, 28] is an operator acting in a tensor square of an $n$-dimensional vector space $V$ and given by

$$
\hat{R}_{\beta_{1} \beta_{2}}^{\alpha_{1} \alpha_{2}}=q^{\delta_{\alpha_{1} \alpha_{2}}} \delta_{\beta_{2}}^{\alpha_{1}} \delta_{\beta_{1}}^{\alpha_{2}}+(q-\bar{q}) \theta_{\alpha_{2} \alpha_{1}} \delta_{\beta_{1}}^{\alpha_{1}} \delta_{\beta_{2}}^{\alpha_{2}}
$$

(no summation in the right hand side) where the indices $\alpha, \beta$ take values from 1 to $n$, and $\theta_{\alpha \beta}=\{1$ if $\alpha>\beta, 0$ if $\alpha \leq \beta\}$.

This $R$-matrix is a particular representative of a family of constant Hecke $R$-matrices, - i.e., it satisfies the braid relation and the Hecke condition

$$
\begin{aligned}
& \hat{R}_{12} \hat{R}_{23} \hat{R}_{12}=\hat{R}_{23} \hat{R}_{12} \hat{R}_{23}, \\
& \hat{R}^{2}=\mathbb{I}+(q-\bar{q}) \hat{R} .
\end{aligned}
$$

Eqs.(2.2), 2.3) imply that the matrices $\hat{R}_{12}, \hat{R}_{23}$ generate a representation of $\mathcal{H}_{3}(q)$ in $V^{\otimes 3}$. For an arbitrary $k$ the representation $\rho_{\hat{R}, k}: \mathcal{H}_{k}(q) \rightarrow$ $\operatorname{Aut}\left(V^{\otimes k}\right)$ generated by a constant Hecke $R$-matrix is defined by

$$
\rho_{\hat{R}, k}\left(g_{i}\right)=\hat{R}_{i i+1}
$$

For representations $\rho_{\hat{R}, k}$ generated by the $R$-matrix (2.1) we have

$$
\text { height } \rho_{\hat{R}, k}=n \quad \text { if } \quad k \geq n .
$$

The rank $1 q$-antisymmetrizers $\rho_{\hat{R}, k}\left(A^{(i, n+i-1)}\right)$ are most conveniently described in terms of $q$-analogues of (co- and contravariant) Levi-Cività tensors which are solutions of the equations

$$
\begin{aligned}
& \hat{R}_{\beta_{i} \beta_{i+1}}^{\alpha_{i} \alpha_{i+1}} \varepsilon^{\alpha_{1} \ldots \beta_{i} \beta_{i+1} \ldots \alpha_{n}}=-\bar{q} \varepsilon^{\alpha_{1} \ldots \alpha_{i} \alpha_{i+1} \ldots \alpha_{n}}, \\
& \varepsilon_{\alpha_{1} \ldots \beta_{i} \beta_{i+1} \ldots \alpha_{n}} \hat{R}_{\alpha_{i} \alpha_{i+1}}^{\beta_{i} \beta_{i+1}}=-\bar{q} \varepsilon_{\alpha_{1} \ldots \alpha_{i} \alpha_{i+1} \ldots \alpha_{n}}, \quad i=1,2, \ldots, n-1 .
\end{aligned}
$$

It is straightforward to prove that these equations have unique (up to normalization) solutions. The rank 1 condition (1.10) follows as a corollary.

\footnotetext{
${ }^{1}$ In general, this need not be the case. Examples with height $\rho \neq \operatorname{dim} V$ were constructed in 25].
} 
In the special case of representations $\rho_{\hat{R}, k}$ generated by the $R$-matrix (2.1) the only nonvanishing components of the $\varepsilon$-tensors have pairwise different indices $\alpha_{1}, \alpha_{2}, \ldots \alpha_{n}$, and can be chosen as

$$
\varepsilon^{\alpha_{1} \alpha_{2} \ldots \alpha_{n}}=\bar{q}^{n(n-1) / 2}(-q)^{\ell(\sigma)}, \quad \varepsilon_{\alpha_{1} \alpha_{2} \ldots \alpha_{n}}=(-q)^{\ell(\sigma)} .
$$

Here $\ell(\sigma)$ is the length of the permutation $\sigma=\left(\begin{array}{c}1,2, \ldots, n \\ \alpha_{1}, \alpha_{2}, \ldots, \alpha_{n}\end{array}\right)$.

The rank $1 q$-antisymmetrizers are expressed in terms of the $\varepsilon$-tensors as

$$
\rho_{\hat{R}, k}\left(A^{(i, n+i-1)}\right)=\frac{1}{[n] !} \varepsilon^{|i \ldots n+i-1\rangle} \varepsilon_{\langle i \ldots n+i-1|}, \quad i=1, \ldots, k-n+1 .
$$

Here (by analogy with the matrix notation) we substitute the vector space indices of $\varepsilon$-tensors by their labels: $\alpha_{i} \rightarrow i$. The "bra" and "ket" notation of $\varepsilon$-tensor indices is used in order to distinguish labels of matrix spaces from those of vector spaces. One should have in mind the following symbolic decomposition for the matrix space label: $i=|i\rangle \otimes\langle i|$. For example, the equation $A_{i} u^{|i\rangle}\left(\equiv A_{\langle i|}^{|i\rangle} u^{|i\rangle}\right)=v^{|i\rangle}$ is to be understood as $\sum_{\beta_{i}} A_{\beta_{i}}^{\alpha_{i}} u^{\beta_{i}}=v^{\alpha_{i}}$.

Finally, we shall adapt for $\rho_{\hat{R}, k}$ those formulas (1.12a-f) which will be used in Section 5. Written in terms of the $\varepsilon$-tensors the relations $(1.12 \mathrm{~b}),(1.12 \mathrm{c})$, (1.12e) and (1.12f) assume the form

$$
\begin{gathered}
\rho_{\hat{R}, n+1}\left(g_{1} \ldots g_{n}\right) \varepsilon^{|1 \ldots n\rangle} \equiv \hat{R}_{12} \ldots \hat{R}_{n n+1} \varepsilon^{|1 \ldots n\rangle}=q \varepsilon^{|2 \ldots n+1\rangle} N_{\langle n+1|}^{|1\rangle} \\
\rho_{\hat{R}, n+1}\left(g_{n} \ldots g_{1}\right) \varepsilon^{|2 \ldots n+1\rangle} \equiv \hat{R}_{n n+1} \ldots \hat{R}_{12} \varepsilon^{|2 \ldots n+1\rangle}=q \varepsilon^{|1 \ldots n\rangle} K^{|n+1\rangle}, \\
K N=N K=\mathbb{I} .
\end{gathered}
$$

Here the matrices $N$ and $K$ are defined as

$$
\begin{aligned}
N_{\langle n+1|}^{|1\rangle} & =\frac{(-1)^{n-1}}{[n-1] !} \varepsilon_{\langle 2 \ldots n+1|} \varepsilon^{|1 \ldots n\rangle}, \\
K_{\langle 1|}^{|n+1\rangle} & =\frac{(-1)^{n-1}}{[n-1] !} \varepsilon_{\langle 1 \ldots n|} \varepsilon^{|2 \ldots n+1\rangle},
\end{aligned}
$$

and for the $\varepsilon$-tensors given by Eq.(2.5) we have just

$$
N=K=\mathbb{I} \text {. }
$$

\section{$3 \quad S L(n)$-type dynamical $R$-matrices}

We now turn to the dynamical $R$-matrix defined in the Introduction. In order to present the QDYBE in a form suitable for our purposes we shall introduce a set of commutative variables

$$
X^{i}, i=1, \ldots, n, \quad\left[X^{i}, X^{j}\right]=0, \quad \prod_{i=1}^{n} X^{i}=1
$$


which play the role of elementary shift operators for $p_{i}$ :

$$
p X^{i}=X^{i}\left(p+v^{(i)}\right) .
$$

The elements $X^{i}$ and $q^{p_{i}}$ provide a realization of (the Weyl's form of) the canonical commutation relations. Note that in concrete applications they can be naturally identified with (a subset of) dynamical variables of a model (see, e.g., [5]).

Let us arrange the auxiliary variables $X^{i}$ into a unimodular diagonal matrix,

$$
X=\operatorname{diag}\left\{X^{1}, \ldots, X^{n}\right\}, \quad \operatorname{det}(X)=1 .
$$

The Hecke-type dynamical R-matrix is characterized by the following set of relations:

$$
\begin{aligned}
\hat{R}_{12}(p)\left(X_{1} \hat{R}_{23}(p) X_{1}^{-1}\right) \hat{R}_{12}(p)= & \left(X_{1} \hat{R}_{23}(p) X_{1}^{-1}\right) \hat{R}_{12}(p)\left(X_{1} \hat{R}_{23}(p) X_{1}^{-1}\right), \\
\hat{R}(p)^{2} & =\mathbb{I}+(q-\bar{q}) \hat{R}(p) \\
\hat{R}_{12}(p) X_{1} X_{2} & =X_{1} X_{2} \hat{R}_{12}(p) .
\end{aligned}
$$

Here the first and second relations are the dynamical Yang-Baxter equation and the Hecke condition, respectively. A condition of type (3.5), although not always imposed on dynamical $R$-matrices, is also necessary in our treatment. As we shall see below, it ensures that conditions (1.3) for the Hecke algebra representations generated by $\hat{R}(p)$ are satisfied.

Following 18 we shall consider dynamical $R$-matrices of the form

$$
\hat{R}_{j_{1} j_{2}}^{i_{1} i_{2}}(p)=a_{i_{1} i_{2}}(p) \delta_{j_{2}}^{i_{1}} \delta_{j_{1}}^{i_{2}}+b_{i_{1} i_{2}}(p) \delta_{j_{1}}^{i_{1}} \delta_{j_{2}}^{i_{2}}, \quad i_{1,2}, j_{1,2}=1, \ldots, n
$$

(there is no summation over repeated upper and lower indices in the right hand side); in order to have a unique decomposition in terms of the unit and the permutation matrices in the tensor square of spaces we impose the condition $b_{i i}(p)=0$. This special class of $p$-dependent Hecke $R$-matrices will be called dynamical $R$-matrices of an $S L(n)$-type $\mathrm{E}$.

The unknown functions $a_{i j}(p), b_{i j}(p)$ in the Ansatz (3.6) are to be fixed by the conditions (3.3-3.5). The Hecke condition (3.4) gives

$$
\begin{gathered}
b_{i i}=0, b_{i j}+b_{j i}=q-\bar{q}, \quad \text { for } i \neq j, \\
a_{i j} a_{j i}-b_{i j} b_{j i}=1, \text { for } i \neq j, \\
a_{i i}^{2}-(q-\bar{q}) a_{i i}=1 .
\end{gathered}
$$

The last equation has two solutions: $a_{i i}= \pm q^{ \pm 1}$ for each $i$. Below we consider only the case $a_{i i}=q, \forall i$ (the other cases correspond, in particular,

\footnotetext{
${ }^{2}$ In $[19$ these $R$-matrices were called $G L(n)$-type $R$-matrices. We call them $S L(n)$ type $R$-matrices instead since we impose the additional condition $\sum_{i=1}^{n} p_{i}=0$ (see (0.2)) on the variables $p_{i}$.
} 
to quantum supergroups and have been considered in [18]). Finally, the dynamical Yang-Baxter equation (3.3) and Eq.(3.5) impose the constraints

$$
\begin{gathered}
a_{i j}\left(p_{1}, \ldots, p_{n}\right)=a_{i j}\left(p_{i j}\right), \quad b_{i j}\left(p_{1}, \ldots, p_{n}\right)=b_{i j}\left(p_{i j}\right), \\
b_{i j} b_{j k} b_{k i}+b_{i k} b_{k j} b_{j i}=0, \\
b_{i j}\left(p_{i j}+1\right)=\frac{b_{i j}\left(p_{i j}\right) q}{\bar{q}+b_{i j}\left(p_{i j}\right)},
\end{gathered}
$$

where $p_{i j}:=p_{i}-p_{j}$. For $a_{i i}=q$ the general solution of (3.7) - 3.11) can be written as 18

$$
a_{i j}(p)=\alpha_{i j}\left(p_{i j}\right) \xi_{i j}\left(p_{i j}\right), \quad b_{i j}(p)=q-\xi_{i j}\left(p_{i j}\right)
$$

where $\xi_{i j}(p)$ are expressed as the following ratios:

$$
\xi_{i j}(p)=\frac{f\left(p_{i j}-1, \beta_{i j}\right)}{f\left(p_{i j}, \beta_{i j}\right)}, \quad f(p, \beta)=\bar{q}^{p}+[p] \beta .
$$

Here $\beta_{i j}\left(p_{i j}\right)=\beta_{i j}\left(p_{i j}+1\right)$. We shall consider $\beta_{i j}$ as constant parameters since their functional dependence does not change any of the results below. The function $f(p, \beta)$ satisfies the finite difference equation

$$
f(p+1, \beta)+f(p-1, \beta)=[2] f(p, \beta)
$$

with the initial conditions

$$
f(0, \beta)=1, \quad f(1, \beta)=\bar{q}+\beta .
$$

Equations (3.14) can be deduced from (3.11).

Not all of the remaining in (3.12), (3.13) parameters $\alpha_{i j}\left(p_{i j}\right)$ and $\beta_{i j}$ are independent. The relations between them are given by

$$
\begin{gathered}
\alpha_{i i}=1, \quad \alpha_{i j}\left(p_{i j}\right) \alpha_{j i}\left(p_{j i}\right)=1, \\
\beta_{i i}=0, \quad \beta_{i j}+\beta_{j i}=q-\bar{q} \quad \text { for } i \neq j, \\
\beta_{i j} \beta_{j k} \beta_{k i}+\beta_{i k} \beta_{k j} \beta_{j i}=0 .
\end{gathered}
$$

An easy way to solve Eqs.(3.17a), (3.17b) is to make the substitution

$$
\beta_{i j}=\frac{q-\bar{q}}{1-\pi_{i j}} \quad \Leftrightarrow \quad \pi_{i j}=\frac{\beta_{i j}-q+\bar{q}}{\beta_{i j}}, \quad \text { for } i \neq j .
$$

We stress that the parameters $\pi_{i i}$ are not fixed here and can be chosen as arbitrary constants. In terms of the new variables $\pi_{i j}$ equations (3.17a), (3.17b) take the simple form

$$
\pi_{i j} \pi_{j i}=1, \quad \pi_{i j} \pi_{j k} \pi_{k i}=1
$$

and are solved by $\pi_{i j}=\prod_{k=i}^{j-1} \pi_{k k+1}=\pi_{j i}^{-1}$, for $i<j$. Hence, 


$$
\beta_{i j}=\frac{(q-\bar{q}) \prod_{k=i}^{j-1} \beta_{k}}{\prod_{k=i}^{j-1} \beta_{k}-\prod_{k=i}^{j-1}\left(\beta_{k}-q+\bar{q}\right)}, \quad \text { for } \quad i<j,
$$

where the remaining $(n-1)$ parameters $\beta_{i} \equiv \beta_{i i+1}$ are independent.

We shall describe two types of transformations on the set of $S L(n)$-type dynamical $R$-matrices.

1. a simple version of twisting for dynamical $R$-matrices:

$$
\hat{R}(p)_{21} \rightarrow{ }^{F} \hat{R}_{21}=\hat{F} \hat{R}(p) \hat{F}^{-1},
$$

where $\hat{F}(p)=F_{12}(p) P_{12}$ and

$$
F_{12} \equiv F_{j_{1} j_{2}}^{i_{1} i_{2}}(p)=\delta_{j_{1}}^{i_{1}} \delta_{j_{2}}^{i_{2}} \psi_{i_{1} i_{2}}(p),
$$

(an analog of Drinfeld's twist, see [29]). An explanations on how this twist works is given in the two Lemmas below.

Lemma 3.1 Let $\hat{F}(p)$ be an operator acting in $V \otimes V$. If

$$
\hat{F}_{12}^{-1} X_{1}^{-1} \hat{F}_{23}=\hat{A}_{123} X_{3}^{-1} \hat{A}_{123}
$$

for some operator $\hat{A}(p)$ acting in $V \otimes V \otimes V$ and

$$
\hat{R}(p)_{12} \hat{A}_{123}=\hat{A}_{123} \hat{R}(p)_{23}
$$

then the matrix ${ }^{F} \hat{R}_{21}=\hat{F} \hat{R}(p) \hat{F}^{-1}$ satisfies (a version of - see the proof) the QDYBE (3.3).

Proof. Substitute ${ }^{F} \hat{R}_{21}$ in the QDYBE taking into account that the QDYBE has two more equivalent forms:

$$
\begin{gathered}
\hat{R}(p)_{23} X_{3}^{-1} \hat{R}(p)_{12} X_{3} \hat{R}(p)_{23}=X_{3}^{-1} \hat{R}(p)_{12} X_{3} \hat{R}(p)_{23} X_{3}^{-1} \hat{R}(p)_{12} X_{3}, \\
\hat{R}(p)_{21} X_{1}^{-1} \hat{R}(p)_{32} X_{1} \hat{R}(p)_{21}=X_{1}^{-1} \hat{R}(p)_{32} X_{1} \hat{R}(p)_{21} X_{1}^{-1} \hat{R}(p)_{32} X_{1} .
\end{gathered}
$$

The first equation results from repeated application of (3.5) to (3.3). The second equation is obtained from the first one by simply permuting the subscripts 1 and 3 .

Lemma 3.2 Let $F_{12}$ be the diagonal matrix (3.20) where $\psi_{i j} \psi_{j i}=1$ and $\psi_{i i}=1$. Assume that $\psi_{i j}$ depends on $p_{i j}$ only. Then, Eqs.(3.21), (3.22) are satisfied by $\hat{A}=A P_{23} P_{12}$ where the matrix $A$ is diagonal, $A_{a b c}^{i j k}=a_{i j k} \delta_{a}^{i} \delta_{b}^{j} \delta_{c}^{k}$, the elements $a_{i j k}$ being given by

$$
a_{i j k}= \begin{cases}\psi_{i k} \psi_{j k} & \text { if } i \neq j \\ {\left[\psi_{i k}\left(p_{i k}+1\right)\right]^{2}} & \text { if } i=j\end{cases}
$$

Proof. The operator $A_{123}$ is symmetric in the first two indices, $A_{123}=$ $A_{213}$ which implies that it commutes with any $R$-matrix of the form (3.6). Therefore, (3.22) is satisfied. 
Eq.(3.21) can be checked directly.

These Lemmas demonstrate that the operator (3.20) indeed generates a twist leading to the changes $\alpha_{i j} \rightarrow \alpha_{i j} \psi_{j i}^{2}, \beta_{i j} \rightarrow \beta_{i j}$ of the parameters in (3.12), (3.13).

2. canonical transformations of the dynamical parameters $p_{i} \rightarrow p_{i}+c_{i}$, where $c_{i}, i=1, \ldots, n$ are arbitrary constants satisfying the condition $\sum_{i=1}^{n} c_{i}=0$.

We conclude the Section by a brief discussion of the structure of the family of $S L(n)$-type dynamical $R$-matrices (3.6), (3.12), (3.13). There are two essentially different domains for the parameters $\beta_{i}$ of this family.

a) $\beta_{i} \neq 0$ and $\beta_{i} \neq q-\bar{q}$, for all $i$.

In this case the whole family (3.6), (3.12), (3.13) can be generated starting from any particular representative with the use of the two types of transformations described above.

Indeed, the parameters $\alpha_{i j}$ can be excluded with the help of a twist. Then, performing a canonical transformation of the form

$$
q^{2 p_{i j}} \rightarrow q^{2 p_{i j}} \pi_{i j}=q^{2 p_{i j}} \prod_{k=i}^{j-1} \frac{\beta_{k}-q+\bar{q}}{\beta_{k}} \text { for } i<j,
$$

for instance, one excludes the parameters $\beta_{i j}$ from the Ansatz (3.6), (3.12), (3.13) and passes to a dynamical $R$-matrix with

$$
\xi_{i j}(p)=\frac{\left[p_{i j}-1\right]}{\left[p_{i j}\right]}
$$

(cf. (3.13) - (3.17b)). This $R$-matrix is the limiting case $\beta_{i} \rightarrow \infty$ of our family, and it is this type of dynamical $R$-matrix which is discussed in [5].

b) Either all $\beta_{i}=q-\bar{q}$, or all $\beta_{i}=0$. We shall consider the first case $\beta_{i}=q-\bar{q}$ for which

$$
\beta_{i j}= \begin{cases}q-\bar{q} & \text { for } \quad i<j \\ 0 & \text { for } \quad i \geq j\end{cases}
$$

and put $\alpha_{i j}\left(p_{i j}\right)=$ const $_{i j}$. In this case the $R$-matrix (3.6), (3.12), (3.13) becomes independent on the dynamical variables $p_{i}$ and is reduced to the constant $R$-matrix describing the multiparametric [30] deformations of $G L(n)$ which are all twist-equivalent.

With the particular choice

$$
\alpha_{i j}=\left\{\begin{array}{lll}
q & \text { for } & i<j \\
1 & \text { for } & i=j \\
\bar{q} & \text { for } & i>j
\end{array}\right.
$$

one reproduces the standard $S L(n)$-type $R$-matrix (2.1).

Remark 3.1 In the intermediate cases (where only a part of the parameters $\beta_{i}$ are equal to 0 or $q-\bar{q}$ ) the corresponding dynamical $R$-matrix $\hat{R}(p)$ contains the (dynamical and constant) $R$-matrices described in $\mathbf{a}$ ) and $\mathbf{b}$ ) as submatrices. 


\section{Representations generated by $S L(n)$-type dynamical $R$-matrices}

Now we are in a position to introduce the Hecke algebra representations associated with Hecke-type dynamical $R$-matrices.

Proposition 4.1 Let $\hat{R}(p)$ be a dynamical R-matrix of the Hecke type. The matrices

$$
\rho_{\hat{R}(p), k}\left(g_{i}\right)=\left(X_{1} X_{2} \ldots X_{i-1}\right) \hat{R}_{i, i+1}(p)\left(X_{1} X_{2} \ldots X_{i-1}\right)^{-1}, i=1, \ldots, k-1,
$$

generate a Hecke algebra representation, $\rho_{\hat{R}(p), k}: \mathcal{H}_{k}(q) \rightarrow \operatorname{Aut}\left(V^{\otimes k}\right)$.

Proof. Obviously, equation (3.3) imply that the matrices $\rho_{\hat{R}(p), k}\left(g_{i}\right)$ and $\rho_{\hat{R}(p), k}\left(g_{i+1}\right)$ satisfy the braid relations (1.1). Then, the conditions (3.5) ensure that the matrices (4.1a) satisfy (1.3) and, therefore, (4.1a) represent the generators of the braid algebra $\mathcal{B}_{k}$. Finally, the Hecke conditions (1.2) for the generators (4.1a) follow from the Hecke property (3.4) of the dynamical $R$-matrix (1.2).

Remark 4.1 In contrast with the case of constant Hecke $R$-matrix (2.4) the representations generated by a dynamical Hecke $R$-matrix are nonlocal; in other words, the matrices $\rho_{\hat{R}(p), k}\left(g_{i}\right)$ act nontrivially as diagonal matrices on $V_{j}$ with $j<i$ (and not merely on $V_{i} \otimes V_{i+1}$ ). Only the representation of the first generator with $i=1$ has the usual 'locality' property.

Remark 4.2 One can construct representations equivalent to $\rho_{\hat{R}(p), k}$ in which some other generator is 'localized' instead $g_{1}$. For instance, the representation $\bar{\rho}_{\hat{R}(p), k}$ which localizes $\bar{\rho}_{\hat{R}(p), k}\left(g_{k-1}\right)$ is given by

$$
\bar{\rho}_{\hat{R}(p), k}(t)=\left(X_{1} X_{2} \ldots X_{k}\right)^{-1} \rho_{\hat{R}(p), k}(t)\left(X_{1} X_{2} \ldots X_{k}\right), \quad \forall t \in \mathcal{H}_{k}(q)
$$

so that

$$
\bar{\rho}_{\hat{R}(p), k}\left(g_{i}\right)=\left(X_{i+2} \ldots X_{k}\right)^{-1} \hat{R}_{i, i+1}(p)\left(X_{i+2} \ldots X_{k}\right) .
$$

Note that in addition to the nonlocal property the representation matrices of $\bar{\rho}_{\hat{R}(p), k}$ depend explicitly on $k$.

From now on we shall restrict ourselves to discussing those representations $\rho_{\hat{R}(p), k}$ which are generated by the $S L(n)$-type dynamical $R$-matrices (3.6), (3.12), (3.13). For $k \geq n$ all these representations are of height $n$. The rank $1 q$-antisymmetrizers are conveniently expressed in terms of dynamical $\mathcal{E}$-tensors $\mathcal{E}^{|1 \ldots n\rangle}(p)$ and $\mathcal{E}_{\langle 1 \ldots n|}(p)$, which are the unique (up to normalization) solutions of the equations

$$
\begin{aligned}
& \rho_{\hat{R}(p), k}\left(g_{i}\right) \mathcal{E}^{|1 \ldots n\rangle}(p)=-\bar{q} \mathcal{E}^{|1 \ldots n\rangle}(p), \quad 1 \leq i \leq n-1 . \\
& \mathcal{E}_{\langle 1 \ldots n|}(p) \rho_{\hat{R}(p), k}\left(g_{i}\right)=-\bar{q} \mathcal{E}_{\langle 1 \ldots n|}(p), \quad
\end{aligned}
$$

The only nonvanishing components of these $\mathcal{E}$-tensors have pairwise different indices $i_{1}, i_{2}, \ldots, i_{n}$ and look like

$$
\mathcal{E}^{i_{1} i_{2} \ldots i_{n}}(p)=(-1)^{\ell(\sigma)} \prod_{(j, i) \in J(\sigma)} \alpha_{j i}\left(p_{j i}\right) \prod_{1 \leq a<b \leq n} \xi_{i_{a} i_{b}}\left(p_{i_{a} i_{b}}\right)
$$




$$
\mathcal{E}_{i_{1} i_{2} \ldots i_{n}}(p)=(-1)^{\ell(\sigma)} \prod_{(j, i) \in J(\sigma)} \alpha_{i j}\left(p_{i j}\right)
$$

Here $\ell(\sigma)$ is the length of the permutation $\sigma=\left(\begin{array}{c}1,2, \ldots, n \\ i_{1}, i_{2}, \ldots, i_{n}\end{array}\right)$, and

$$
J(\sigma)=\left\{\left(i_{a}, i_{b}\right): a<b, i_{a}>i_{b}\right\} .
$$

The dynamical $\mathcal{E}$-tensors (4.3), (4.4) are normalized so that they would coincide with the constant $\varepsilon$-tensors (2.5) in the case (3.25), (3.26).

Now the expressions for rank $1 q$-antisymmetrizers in the representations $\rho_{\hat{R}(p), k}$ are given by

$$
\begin{aligned}
& \rho_{\hat{R}(p), k}\left(A^{(i, n+i-1)}\right)= \\
& \quad \frac{1}{[n] !}\left(X_{1} \ldots X_{i-1}\right) \mathcal{E}^{|i \ldots n+i-1\rangle}(p) \mathcal{E}_{\langle i \ldots n+i-1|}(p)\left(X_{1} \ldots X_{i-1}\right)^{-1} .
\end{aligned}
$$

The numerical coefficient in this formula is calculated with the use of the relation

$$
\mathcal{E}_{\langle 1 \ldots n|}(p) \mathcal{E}^{|1 \ldots n\rangle}(p)=[n] !
$$

which is proved in the Appendix.

We conclude the discussion on dynamical $R$-matrices by writing down formulas $(1.12 \mathrm{a}),(1.12 \mathrm{~d})$, and $(1.12 \mathrm{e}),(1.12 \mathrm{f})$ for the representation $\rho_{\hat{R}(p), n+1}$ :

$$
\begin{gathered}
\mathcal{E}_{\langle 1 \ldots n|}(p) \rho_{\hat{R}(p), n+1}\left(g_{n} g_{n-1} \ldots g_{1}\right)=q K_{\langle 1|}^{|n+1\rangle}(p) X_{1} \mathcal{E}_{\langle 2 \ldots n+1|}(p) X_{1}^{-1} \\
X_{1} \mathcal{E}_{\langle 2 \ldots n+1|}(p) X_{1}^{-1} \rho_{\hat{R}(p), n+1}\left(g_{1} g_{2} \ldots g_{n}\right)=q N_{\langle n+1|}^{|1\rangle}(p) \mathcal{E}_{\langle 1 \ldots n|}(p) \\
K(p) N(p)=N(p) K(p)=\mathbb{I}
\end{gathered}
$$

Here the matrices $N(p), K(p)$ are defined as

$$
\begin{aligned}
N_{\langle n+1|}^{|1\rangle}(p) & =\frac{(-1)^{n-1}}{[n-1] !} X_{1} \mathcal{E}_{\langle 2 \ldots n+1|}(p) X_{1}^{-1} \mathcal{E}^{|1 \ldots n\rangle}(p) \\
K_{\langle 1|}^{|n+1\rangle}(p) & =\frac{(-1)^{n-1}}{[n-1] !} \mathcal{E}_{\langle 1 \ldots n|}(p) X_{1} \mathcal{E}^{|2 \ldots n+1\rangle}(p) X_{1}^{-1} .
\end{aligned}
$$

For the $S L(n)$-type dynamical $R$-matrices the matrices $N(p), K(p)$ are diagonal. Inserting formulas (4.3), (4.4) for the dynamical $\varepsilon$-tensors into (4.10), (4.11) and using (4.6), one ends up with the following expressions for their diagonal components:

$$
N_{i}^{i}(p)=\left(K_{i}^{i}(p)\right)^{-1}=\prod_{j \neq i} \alpha_{i j}\left(p_{i j}-\theta_{j i}\right) \xi_{i j}\left(p_{i j}\right)
$$

where $\theta_{j i}=\{1$ if $j>i, 0$ if $j<i\}$. 


\section{$5 \quad$ Quantum matrix algebra $\mathcal{A}(\hat{R}(p), \hat{R})$ : quan- tum determinant and inversion formula}

We shall apply the above technique to the quantum matrix algebra $\mathcal{A}$ which is defined as follows (cf. Introduction).

Definition 5.1 Let $\mathbb{F}$ be the field of the complex meromorphic functions of the (commuting) variables $p_{j}, j=1, \ldots, n$. Let $\hat{R}(p)$ be a dynamical $R$ matrix of an $S L(n)$-type and $\hat{R}$ be a constant $S L(m)$-type $R$-matrix. Assume that both $\hat{R}(p)$ and $\hat{R}$ satisfy the Hecke condition (0.4) with the same value of $q$. Then $\mathcal{A}=\mathcal{A}(\hat{R}(p), \hat{R})$ is a complex algebra with 1 that is generated by $\mathbb{F}$ and the $m n$ elements $a_{\alpha}^{i}(i=1, \ldots, n$ and $\alpha=1, \ldots, m)$, satisfying the relations

$$
\begin{aligned}
& \hat{R}(p)_{12} a_{1} a_{2}=a_{1} a_{2} \hat{R}_{12}, \\
& a f(p)=X f(p) X^{-1} a, \quad \forall f(p) \in \mathbb{F},
\end{aligned}
$$

where $X$ is a unimodular diagonal matrix (3.2) whose diagonal elements $X^{i}$ satisfy (3.1a), (3.1b).

Remark 5.1 The definition above is given for arbitrary $m$ and $n$. However in the sequel we shall discuss the case $m=n$ only.

Remark 5.2 For the applications envisaged here, the field $\mathbb{F}$ of meromorphic functions of $p_{j}$ can be replaced by its subfield of rational functions of $q^{p_{j}}$ (as stated in the Introduction). Then we should just require

$$
X^{k} q^{p_{i j}}\left(X^{k}\right)^{-1}=q^{p_{i j}+\delta_{j k}-\delta_{i k}}
$$

instead of (3.1b). Note that for $q$ a root of unity, $q^{2 h}=1$ (cf. Eq.(6.8) below) $p_{i j}$ are only determined up to an additive integer multiple of $2 h$.

Remark 5.3 More general matrix algebras are of interest in which the $R$ matrices on both sides of the quadratic relations (5.1) are allowed to depend on possibly different sets of commuting variables $p$ and $p^{\prime}$

$$
\hat{R}(p)_{12} Q_{1} Q_{2}=Q_{1} Q_{2} \hat{R}^{\prime}\left(p^{\prime}\right)_{12},
$$

while the shift properties assume the form

$$
p_{k l} Q_{j}^{i}=Q_{j}^{i}\left(p_{k l}+\delta_{k}^{i}-\delta_{l}^{i}\right) \quad, \quad p_{k l}^{\prime} Q_{j}^{i}=Q_{j}^{i}\left(p_{k l}^{\prime}+\delta_{j k}-\delta_{j l}\right) .
$$

Such $\mathcal{A}\left(\hat{R}(p), \hat{R}^{\prime}\left(p^{\prime}\right)\right)$ can be treated in much the same way or reduced to the study of two matrix algebras of the above type setting $Q_{j}^{i}=a_{\alpha}^{i} \bar{a}_{j}^{\alpha}$, where $a$ and $\bar{a}$ satisfy exchange relations of the type (0.6) and (0.10), respectively (see [24]). Note that dynamical quantum groups (introduced in [16]) are defined by relations similar to (5.3) and (5.4) but with the dynamical $R$ - matrices (and momenta $p, p^{\prime}$ ) related to each other by some equivalence transformation $\hat{R}^{\prime}\left(p^{\prime}\right)=X^{-1} \hat{R}(p) X$. Another desirable modification of the matrix algebra (5.1) corresponds to the case when $\hat{R}$ is an $S O_{q}$ or $S p_{q}$ constant $R$-matrix. 
In this case $\hat{R}$ and $\hat{R}(p)$ satisfy a third order (Birman-Wenzl) condition instead of the Hecke property (1.2) and the QDYBE (3.3) have to be modified correspondingly.

Remark 5.4 The algebra $\mathcal{A}$ differs from the one considered in 33 where the counterpart of a matrix $b=X^{-1} a$ (denoted by $u_{\alpha}^{i}(\bar{\omega})$ in Eq.(11) of [33]) which commutes with $p$ is used for changing the basis of chiral vertex operators. It is assumed in 33 that the elements of $b$ only depend on $p$ and hence commute among themselves while in our case this is not so. Indeed, the reflection equation subalgebra $\mathcal{M}(\hat{R})$, defined in Proposition 5.5 below, is non-commutative although its elements commute with the $p$ 's. The difference is essential: as a result, Cremmer and Gervais do not recover the standard (constant) $S L_{q}(n) R$-matrix for $n>2$ but introduce instead new solutions of the Yang-Baxter equation. One of the authors (I.T.) would like to thank J.-L. Gervais and E. Cremmer for an enlightening discussion on this point.

The term "matrix algebra" for the algebra $\mathcal{A} \equiv \mathcal{A}(\hat{R}(p), \hat{R})$ is justified by the fact that we shall be able to (define and) compute the determinant of $a-$ as a function of $p-$ and to find the inverse of $a$. In the case of $2 \times 2$ matrices the determinant of $a$ was constructed in [4] (see also [32]) for the special choice $\beta_{i} \rightarrow \infty, \alpha_{i j}=1$ of the parameters. We shall present the definition of the determinant in a general setting.

Definition 5.2 Let $a=\left\|a_{\alpha}^{i}\right\|$ be the matrix of generators of the algebra $\mathcal{A}(\hat{R}(p), \hat{R})$. The determinant of the matrix a is given by

$$
\operatorname{det}(a)=\frac{1}{[n] !} \mathcal{E}_{\langle 1 \ldots n|}(p) a_{1} a_{2} \ldots a_{n} \varepsilon^{|1 \ldots n\rangle} .
$$

The meaning of this definition is made clear by the following three Propositions. The first and the third of them are the quantum analogues of the basic determinant properties. The second one allows to perform an $S L(n)$ reduction in the algebra $\mathcal{A}(\hat{R}(p), \hat{R})$.

Proposition 5.1 The product $\left(a_{1} a_{2} \ldots a_{n}\right)$ intertwines between constant and dynamical $\varepsilon$-tensors:

$$
\begin{aligned}
\mathcal{E}_{\langle 1 \ldots n|}(p) a_{1} a_{2} \ldots a_{n} & =\operatorname{det}(a) \varepsilon_{\langle 1 \ldots n|} \\
a_{1} a_{2} \ldots a_{n} \varepsilon^{|1 \ldots n\rangle} & =\mathcal{E}^{|1 \ldots n\rangle}(p) \operatorname{det}(a)
\end{aligned}
$$

Proof. First, observe that due to the relations (5.1), (5.2) the product of $k$ matrices $\left(a_{1} a_{2} \ldots a_{k}\right)$ intertwines between the representations $\rho_{\hat{R}, k}$ and $\rho_{\hat{R}(p), k}$ of the algebra $\mathcal{H}_{k}(q)$. Indeed,

$$
\begin{aligned}
& \left(a_{1} \ldots a_{k}\right) \rho_{\hat{R}, k}\left(g_{i}\right)=\left(a_{1} \ldots a_{k}\right) \hat{R}_{i(i+1)} \\
= & a_{1} \ldots a_{i-1}\left(\hat{R}_{i(i+1)}(p) a_{i} a_{i+1}\right) a_{i+2} \ldots a_{k} \\
= & \left(X_{1} \ldots X_{i-1}\right) \hat{R}_{i(i+1)}(p)\left(X_{1} \ldots X_{i-1}\right)^{-1}\left(a_{1} \ldots a_{k}\right)=\rho_{\hat{R}(p), k}\left(g_{i}\right)\left(a_{1} \ldots a_{k}\right) .
\end{aligned}
$$

\footnotetext{
${ }^{3}$ More conventional quantum matrix algebras have been introduced in [1, 30] and their matrix nature were further investigated in [25, 31.
} 
In particular, one has

$$
\left(a_{1} \ldots a_{n}\right) \rho_{\hat{R}, n}\left(A^{(n)}\right)=\rho_{\hat{R}(p), n}\left(A^{(n)}\right)\left(a_{1} \ldots a_{n}\right) .
$$

Multiplying both sides by $\rho_{\hat{R}, n}\left(A^{(n)}\right)$ from the right or by $\rho_{\hat{R}, n}\left(A^{(n)}\right)$ from the left and using projector property of the $q$-antisymmetrizer one comes to the equations

$$
\begin{aligned}
\rho_{\hat{R}(p), n}\left(A^{(n)}\right)\left(a_{1} \ldots a_{n}\right) & =\rho_{\hat{R}(p), n}\left(A^{(n)}\right)\left(a_{1} \ldots a_{n}\right) \rho_{\hat{R}, n}\left(A^{(n)}\right) \\
\left(a_{1} \ldots a_{n}\right) \rho_{\hat{R}, n}\left(A^{(n)}\right) & =\rho_{\hat{R}(p), n}\left(A^{(n)}\right)\left(a_{1} \ldots a_{n}\right) \rho_{\hat{R}, n}\left(A^{(n)}\right) .
\end{aligned}
$$

Finally, expressing (2.6), (4.5) for constant and dynamical $q$-antisymmetrizers in terms of the $\varepsilon$-tensors, one transforms (5.9), (5.10) to the form (5.6), (5.7).

Proposition 5.2 The element $\operatorname{det}(a)$ of the algebra $\mathcal{A}(\hat{R}(p), \hat{R})$ commutes with the generators $p_{i}$ and its commutation with the generators $a_{\alpha}^{i}$ is described by

$$
\operatorname{det}(a) a=K(p) a \operatorname{det}(a),
$$

where the diagonal matrix $K(p)$ is given in ( 4.11 ), ( 4.19 ).

Proof. Consider the permutation of $\operatorname{det}(a)$ with an arbitrary function $h(p)$ :

$$
\begin{aligned}
& \operatorname{det}(a) h(p)=\mathcal{E}_{\langle 1 \ldots n|}(p) a_{1} \ldots a_{n} h(p) \varepsilon^{|1 \ldots n\rangle} /[n] ! \\
& \quad=\mathcal{E}_{\langle 1 \ldots n|}(p)\left(X_{n} \ldots X_{1}\right) h(p)\left(X_{n} \ldots X_{1}\right)^{-1} a_{1} \ldots a_{n} \varepsilon^{|1 \ldots n\rangle} /[n] !
\end{aligned}
$$

Since the only nonvanishing components of the tensor $\mathcal{E}_{\langle 1 \ldots n|}(p)$ are those with pairwise different indices and due to the diagonal structure of the matrix $X$ one has

$$
\mathcal{E}_{\langle 1 \ldots n|}(p) X_{n} \ldots X_{1}=\mathcal{E}_{\langle 1 \ldots n|}(p) \operatorname{det}(X)=\mathcal{E}_{\langle 1 \ldots n|}(p),
$$

where in the last equality the unimodularity of $X$ (see (3.2)) is taken into account. Now we can complete the transformation of (5.12):

$$
\begin{gathered}
\operatorname{det}(a) h(p)=\ldots \\
=h(p) \mathcal{E}_{\langle 1 \ldots n|}(p)\left(X_{n} \ldots X_{1}\right)^{-1} a_{1} \ldots a_{n} \varepsilon^{|1 \ldots n\rangle} /[n] !=h(p) \operatorname{det}(a) .
\end{gathered}
$$

This proves commutativity of $\operatorname{det}(a)$ and $p_{i}$.

Consider now permutation of $\operatorname{det}(a)$ with the matrix $a$. It is technically convenient to take $a$ living in the matrix space with label $(n+1)$ :

$$
\begin{aligned}
\operatorname{det} & (a) a_{n+1}=\mathcal{E}_{\langle 1 \ldots n|}(p)\left\{a_{1} \ldots a_{n} a_{n+1}\right\} \varepsilon^{|1 \ldots n\rangle} /[n] ! \\
& =\left\{\mathcal{E}_{\langle 1 \ldots n|}(p) \rho_{\hat{R}(p), n+1}\left(g_{n} \ldots g_{1}\right)\right\} a_{1} \ldots a_{n+1}\left\{\rho_{\hat{R}, n+1}^{-1}\left(g_{n} \ldots g_{1}\right) \varepsilon^{|1 \ldots n\rangle}\right\} /[n] ! \\
& =K^{|n+1\rangle}(p)\left\{X_{1} \mathcal{E}_{\langle 2 \ldots n+1|}(p) X_{1}^{-1} a_{1}\right\}\left\{a_{2} \ldots a_{n+1} \varepsilon^{|2 \ldots n+1\rangle}\right\} K^{-1|1\rangle}\langle n+1| /[n] ! \\
& =K^{|n+1\rangle}\langle 1| \\
& =(K) a_{1}\left\{\mathcal{E}_{\langle 2 \ldots n+1|}(p) \mathcal{E}^{|2 \ldots n+1\rangle}(p)\right\} \operatorname{det}(a) K_{\langle n+1|}^{-1|1\rangle} /[n] ! \\
& \left(K(p) a K^{-1}\right)_{n+1} \operatorname{det}(a) .
\end{aligned}
$$


The following formulae are used in the course of the calculation: (5.5) and (5.8) in the first line, (4.7) and (2.8) in the second line, (5.2) and (5.7) in the third line, and (4.6) in passing to the last line. For clarity we put into braces those expressions which are to be transformed in the next step.

Finally, substituting $\mathbb{I}$ for the matrix $K$ (see (2.12)) we obtain (5.11).

Corollary 5.1 The element

$$
\Delta=\operatorname{det}(a) \prod_{i<j} \frac{\varphi_{i j}\left(p_{i j}\right)}{f\left(p_{i j}\right)},
$$

where $f\left(p_{i j}\right)=\bar{q}^{p_{i j}}+\left[p_{i j}\right] \beta_{i j}$ and the functions $\varphi_{i j}$ are defined by the relations

$$
\alpha_{i j}\left(p_{i j}\right)=\frac{\varphi_{i j}\left(p_{i j}+1\right)}{\varphi_{i j}\left(p_{i j}\right)}
$$

belongs to the center of the algebra $\mathcal{A}(\hat{R}(p), \hat{R})$. The $S L(n)$-reduction in the algebra $\mathcal{A}(\hat{R}(p), \hat{R})$ can be performed by imposing the condition $\Delta=1$.

Proof. We shall search for the central element in $\mathcal{A}(\hat{R}(p), \hat{R})$ in the form $\Delta=U(p) \operatorname{det}(a)$, where $U(p)$ is some function of $p_{i}$ which is to be fixed. As follows from the Proposition 5.2 the element $\Delta$ commutes with $p_{i}$ and its commutativity with the generators $a_{\alpha}^{i}$ imposes the following conditions on the function $U$

$$
X^{i} U(p)\left(X^{i}\right)^{-1}=U(p) K_{i}^{i}(p), \quad i=1, \ldots, n .
$$

Now using (3.1b), (3.13) and (4.12) it is straightforward to check that with the choice (5.13), (5.14) one satisfies conditions (5.15).

Proposition 5.3 Let the algebra $\mathcal{A}(\hat{R}(p), \hat{R})$ be completed with the inverse determinant of $a:(\operatorname{det} a)^{-1} \operatorname{det}(a)=\operatorname{det}(a)(\operatorname{det} a)^{-1}=1$. Then the left and right inverse of $a$ is given by

$$
\left(a^{-1}\right)_{\langle n+1|}^{|1\rangle}=\frac{(-1)^{n-1}}{[n-1] !}(\operatorname{det} a)^{-1} \mathcal{E}_{\langle 2 \ldots n+1|}(p) a_{2} \ldots a_{n} \varepsilon^{|1 \ldots n\rangle} .
$$

Proof. We first check that the expression (5.16) is a left inverse of $a$ :

$$
\begin{gathered}
\left(a^{-1}\right)^{|1\rangle}{ }_{\langle n+1|} a_{n+1}=\frac{(-1)^{n-1}}{[n-1] !}(\operatorname{det} a)^{-1}\left\{\mathcal{E}_{\langle 2 \ldots n+1|}(p) a_{2} \ldots a_{n} a_{n+1}\right\} \varepsilon^{|1 \ldots n\rangle} \\
=\frac{(-1)^{n-1}}{[n-1] !}(\operatorname{det} a)^{-1} \operatorname{det}(a) \varepsilon_{\langle 2 \ldots n+1|} \varepsilon^{|1 \ldots n\rangle}=N_{\langle n+1|}^{|1\rangle}=\mathbb{I}_{\langle n+1|}^{|1\rangle} .
\end{gathered}
$$

Here we have used successively Eqs.(5.6), (2.10) and (2.12).

Checking that $(5.16)$ is also a right inverse is slightly more complicated:

$$
\begin{aligned}
& a_{1}\left(a^{-1}\right)_{\langle n+1|}^{|1\rangle}=\frac{(-1)^{n-1}}{[n-1] !}\left\{a_{1}(\operatorname{det} a)^{-1}\right\} \mathcal{E}_{\langle 2 \ldots n+1|}(p) a_{2} \ldots a_{n} \varepsilon^{|1 \ldots n\rangle} \\
& =\frac{(-1)^{n-1}}{[n-1] !}(\operatorname{det} a)^{-1} K_{1}(p)\left\{a_{1} \mathcal{E}_{\langle 2 \ldots n+1|}(p)\right\} a_{2} \ldots a_{n} \varepsilon^{|1 \ldots n\rangle} \\
& =\frac{(-1)^{n-1}}{[n-1] !}(\operatorname{det} a)^{-1} K_{1}(p) X_{1} \mathcal{E}_{\langle 2 \ldots n+1|}(p) X_{1}^{-1}\left\{a_{1} \ldots a_{n} \varepsilon^{|1 \ldots n\rangle}\right\} \\
& =(\operatorname{det} a)^{-1} K_{1}(p) N_{\langle n+1|}^{|1\rangle}(p) \operatorname{det}(a)=\mathbb{I}_{\langle n+1|}^{|1\rangle},
\end{aligned}
$$


where we have applied successively Eqs.(5.11), (5.2), (5.7), (4.10) and (4.9).

The existence of inverse matrix $a^{-1}$ is needed in many applications of the algebra $\mathcal{A}(\hat{R}(p), \hat{R})$. As an example of such application we shall construct a realization of a reflection equation algebra $\mathcal{M}(\hat{R})$ (for definition of this algebra see e.g. [34 and references therein) in terms of the generators of $\mathcal{A}(\hat{R}(p), \hat{R})$. We have to use here the following general property of $S L(n)$ type dynamical $R$-matrices (which has been noticed in [5] for the $S L(2)$ case, see also [4], [14]):

Proposition 5.4 The dynamical matrix $\hat{R}(p)$ (3.6), (3.19), (3.13) satisfies the equation

$$
D_{1} \hat{R}(p) D_{2}^{-1}=\hat{R}(p)^{-1} \sigma_{12},
$$

where the diagonal matrices $D$ and $\sigma$

$$
D_{j}^{i} \equiv q^{d_{i}} \delta_{j}^{i}, \quad\left(\sigma_{12}\right)_{j_{1} j_{2}}^{i_{1} i_{2}}=\delta_{j_{1}}^{i_{1}} \delta_{j_{2}}^{i_{2}} \sigma_{i_{1} i_{2}}
$$

are fixed by $(5.17)$ as

$$
\begin{gathered}
q^{d_{i}-d_{j}}=q^{-2 p_{i j}} \pi_{i j} \quad(i \neq j), \\
\sigma_{i j}=q^{2 \delta_{i j}}
\end{gathered}
$$

so that $d_{i}$ are functions of $p$.

Proof. First of all we note that from the Hecke condition (3.4) (and (3.7)) one can deduce

$$
\hat{R}(p)^{-1}=\left(a_{i_{1} i_{2}}(p)-(q-\bar{q}) \delta_{i_{1} i_{2}}\right) \delta_{j_{2}}^{i_{1}} \delta_{j_{1}}^{i_{2}}-b_{i_{2} i_{1}}(p) \delta_{j_{1}}^{i_{1}} \delta_{j_{2}}^{i_{2}}
$$

Substitution of $(3.6)$, (5.18) and (5.21) into (5.17) gives the following equations for the parameters $\sigma_{i j}$ and $d_{i}$

$$
\begin{gathered}
a_{i j}=\left(a_{i j}-(q-\bar{q}) \delta_{i j}\right) \sigma_{j i}, \\
q^{d_{i}-d_{j}} b_{i j}=-b_{j i} \sigma_{i j} .
\end{gathered}
$$

Equation (5.22) leads to (5.20) while (5.23) is equivalent (in view of (A.7)) to (5.19).

Now we construct the matrix $M^{\alpha}{ }_{\beta}$ which is diagonalized with the help of the matrix $a_{\alpha}^{i}$ and the spectrum of which is defined by the matrix $D$ (5.18), (5.19)

$$
M=a^{-1} D a .
$$

It is clear that $\left[D_{1}, D_{2}\right]=0$ and therefore the spectrum of the matrix $M$ gives a commutative set of elements.

Proposition 5.5 The elements of the matrix $M$ (5.24) satisfy a reflection equation of the form

$$
M_{2} \hat{R}^{-1} M_{2} \hat{R}^{-1}=\hat{R}^{-1} M_{2} \hat{R}^{-1} M_{2},
$$


and thus provide a realization of a reflection equation subalgebra $\mathcal{M}(\hat{R})$ in $\mathcal{A}(\hat{R}(p), \hat{R})$. The matrix elements of $M$ satisfy the following exchange relations with the generators of $\mathcal{A}(\hat{R}(p), \hat{R})$ :

$$
\left[D_{2}, M_{1}\right]=0, \quad M_{1} a_{2}=q^{2 / n} a_{2} \hat{R}^{-1} M_{2} \hat{R}^{-1} .
$$

Proof. Using (5.2), one can bring the commutation relations of the matrix $D$ with the elements $a_{\alpha}^{i}$ to the form

$$
a_{1} D_{2}=q^{-2 / n} \sigma_{12} D_{2} a_{1}
$$

where the diagonal matrix $\sigma_{12}$ is given by (5.18), (5.20). Eqs.(5.24) and

(5.27) imply $\left[D_{2}, M_{1}\right]=0$. Then one proves (5.25) and the second relation in (5.26) by using (5.24), (5.1), (5.27) and (5.17) .

\section{Application to the $S U(n)$ WZNW model}

As an application of quantum matrix algebras we briefly describe here a typical problem of the two dimensional conformal field theory in which such matrices arise (see [24] for more details).

Let $G$ be a connected compact Lie group and $g=g(t, x)$ be a map from the cylinder $\mathbb{R} \times \mathbb{S}^{1}$ into $G$ which satisfies the Wess-Zumino-Novikov-Witten (WZNW) equations of motion. The general periodic solution $g(t, x)=$ $g(t, x+2 \pi)$ of these equations factorizes into a product of group valued chiral fields

$$
g_{B}^{A}(t, x)=u_{\alpha}^{A}(x-t) \bar{u}_{B}^{\alpha}(x+t) \quad(\text { classically, } g, u, \bar{u} \in G),
$$

each of which satisfies a twisted periodicity condition; in particular,

$$
u(x+2 \pi)=u(x) M, \quad(M \in G)
$$

where $M$ is the monodromy.

Furthermore, the quantum chiral fields obey quadratic exchange relations 35, 6, 10, 11, 5, 23, 36, 20, 21

$$
u(y)_{2} u(x)_{1}=u(x)_{1} u(y)_{2} R(x-y) \Leftrightarrow P u(y)_{2} u(x)_{1}=u(x)_{2} u(y)_{1} \hat{R}(x-y) .
$$

Here the matrix $R(x)$ is a solution of the the quantum Yang-Baxter equation

whose $x$-dependence is given by a step function, while $\hat{R}(x)$ is the associated braid operator:

$$
\begin{gathered}
\hat{R}(x)=\hat{R} \theta(x)+\hat{R}^{-1} \theta(-x), \quad \hat{R}(x)=P R(x)=\hat{R}^{\varepsilon(x)} \\
(\varepsilon(x)=\theta(x)-\theta(-x)) .
\end{gathered}
$$


Since $\hat{R}$ enters Eq. (6.3) in pair with $P$ it should be normalized to have determinant $\operatorname{det} \hat{R}=\operatorname{det} P$. For $G=S U(n)$ this implies the relation

$$
\hat{R}_{i i+1}=\bar{q}^{\frac{1}{n}} \rho\left(g_{i}\right)\left(\text { for } g_{i}^{2}=\mathbb{I}+(q-\bar{q}) g_{i}\right) \Longrightarrow \operatorname{det} \hat{R}=\operatorname{det} P=(-1)^{\left(\begin{array}{l}
n \\
2
\end{array}\right)}
$$

so that we have to renormalize $\hat{R}$ of $(2.1)$ by multiplying it by $\bar{q}^{\frac{1}{n}}$. (The resulting $\hat{R}$ has eigenvalues $q^{1-\frac{1}{n}}$ and $-\bar{q}^{1+\frac{1}{n}}$ of multiplicities $\left(\begin{array}{c}n+1 \\ 2\end{array}\right)$ and $\left(\begin{array}{c}n \\ 2\end{array}\right)$, respectively; thus the product of all $n^{2}$ eigenvalues of $\hat{R}$ is indeed $(-1)^{\left(\begin{array}{c}n \\ 2\end{array}\right)}$.)

We expand, following [21] and [⿴囗. $u(x)$ into a basis of zero modes that diagonalizes the monodromy matrix $M$ :

$$
u_{\alpha}^{A}(x)=a_{\alpha}^{i} u_{i}^{A}(x, p), \quad a M=D a, \quad D_{j}^{i}=q^{d_{i}} \delta_{j}^{i} .
$$

Here $d_{i}=-2 p_{i}-1 / n+1, p=\left\{p_{i}\right\}$ are central elements of the reflection equation algebra $\mathcal{M}(\hat{R})$; in the quantum field theoretic representation at hand they form a commuting set of operators such that Eq.(0.7) takes place. The eigenvalues of the differences $p_{i i+1}\left(=p_{i}-p_{i+1}\right)$ are natural numbers that can be identified with the extended weights, $\lambda_{i}+1$ labeling the (finite dimensional) irreducible representations of $S U(n)$. The labels of the $\left(\begin{array}{c}n \\ j\end{array}\right)$ dimensional fundamental representation are given by $\lambda_{i}^{(j)}=\delta_{i}^{j}, 1 \leq i, j \leq$ $n-1$. Under these assumptions Eq.(6.3) implies exchange relations of the type

$$
\tilde{R}(p) a_{2} a_{1}=a_{2} a_{1} \hat{R}
$$

where $\tilde{R}(p)$ obeys a QDYBE analogous to (0.3). Hence, the results displayed in Sections 3 and 4 can be applied with slight modifications. (Since $\hat{R}$ and $\tilde{R}(p)$ enter 6.7) homogeneously, the factor $\bar{q}^{\frac{1}{n}}$ of (6.5) cancels in the two sides). Thus we can also apply the results of Section 5 to the (chiral zero mode) quantum matrix algebra $\mathcal{A}$ of the $S U(n)$ WZNW model. It should be noted that in this case $q$ is a root of -1 associated with the level $k \widehat{s u}(n)$ Kac-Moody algebra:

$$
q=e^{i \frac{\pi}{h}}, \quad[2]=q+\bar{q}=2 \cos \frac{\pi}{h}, \quad h=n+k(\geq n+1) .
$$

The eigenvalues $q^{d_{i}}$ of the diagonal matrix $D$ can be expressed as differences of conformal dimensions. Indeed, according to [21], the chiral vertex operators $u_{j}(x, p)$ satisfy

$$
u_{j}(x+2 \pi, p)=u_{j}(x, p) e^{2 \pi i\left(\Delta_{h}(p)-\Delta_{h}\left(p+v^{(j)}\right)\right)},
$$

where the matrices $v^{(j)}$ and $p$ are defined by $(0.1)$ and $(0.2)$. Here the conformal dimensions are expressed in terms of the $S U(n)$ Casimir operator,

$$
2 h \Delta_{h}(p)=C_{2}(p)=\frac{1}{n} \sum_{1 \leq i<k \leq n} p_{i k}^{2}-\frac{n\left(n^{2}-1\right)}{12}
$$

so that

$$
d_{j}=C_{2}(p)-C_{2}\left(p+v^{(j)}\right)=-2\left(p \mid v^{(j)}\right)-\left|v^{(j)}\right|^{2}=\frac{1}{n}-1-2 p_{j} .
$$


(cf. (5.19)).

An important consequence of (6.7) and (6.8) is the existence of an ideal $\mathcal{I}_{h}$ of $\mathcal{A}$ generated by $n^{2}$ elements $\left(a_{\alpha}^{i}\right)^{h}$ such that the factor algebra $\mathcal{A} / \mathcal{I}_{h}$ is finite dimensional [24]. This allows to define a finite dimensional "Fock space representation" of $\mathcal{A}$ with a unique vacuum vector $\mid$ vac $>$ corresponding to trivial $\operatorname{su}(n)$ weight $\lambda_{i}=0\left(p_{i i+1}=1, i=1, \ldots, n-1\right)$ such that

$$
a_{\alpha}^{i} \mid \text { vac }>=0 \quad \text { for } i>1, \quad \mathcal{I}_{h} \mid \text { vac }>=0 .
$$

\section{Acknowledgements}

This work started while three of us, L.K.H., O.V.O. and I.T.T. were visiting the Bogoliubov Laboratory of Theoretical Physics of the JINR, Dubna and was completed while A.P.I., L.K.H. and I.T.T. were visiting the Department of Physics of the University of Pisa, ICTP (Trieste) and the Erwin Schrödinger Institute for Mathematical Physics, respectively. We thank all these Institutions for hospitality and support. This work was also supported in part by RFBR (grant 97-01-01041), INTAS (grant 93-127-ext), by the exchange program between INFN and JINR (Dubna) and by the Bulgarian National Foundation for Scientific Research under contract F-404.

\section{Appendix. Normalization of dynamical Levi- Cività tensors}

The definitions (4.3), (4.4) lead to the expression

$$
\mathcal{E}_{i_{1} \ldots i_{n}}(p) \mathcal{E}^{i_{1} \ldots i_{n}}(p)=\prod_{1 \leq a<b \leq n} \xi_{i_{a} i_{b}}
$$

(there are no summations over the indices $i_{k}$ ), and the normalization condition (14.6) for the dynamical $\mathcal{E}$-tensors follows from

Proposition A Let $\xi_{i j}=d-b_{i j}$ where $d$ is a constant (comparing with (3.19), one gets $d=q$ ) and the elements $b_{i j}$ satisfy (3.7), (3.19). Then the following identity holds:

$$
I_{k} \equiv \sum_{S_{k}} \prod_{1 \leq a<b \leq k} \xi_{i_{a} i_{b}}=[k]_{d} !,
$$

where $k \leq n,[k]_{d}=\frac{\left(d^{k}-(d-\lambda)^{k}\right)}{\lambda} \quad(\lambda=q-\bar{q})$ and $S_{k}$ denotes all permutations of indeces $\left(i_{1}, \ldots, i_{k}\right)\left(i_{a} \neq i_{b}\right.$ for $\left.a \neq b\right)$. Note that $[k]_{d}=[k]$ for $d=q$ (as it is needed in (4.6)).

Proof We shall proceed by induction. For $k=2$ we have

$$
I_{2}=\xi_{i_{1} i_{2}}+\xi_{i_{2} i_{1}}=2 d-\lambda=[2]_{d} .
$$


Let (A.2) is correct for some $k(1<k<n)$, then for $k+1$ we derive

$$
\begin{gathered}
I_{k+1}=\sum_{S_{k+1}}\left[\left(\prod_{l=1}^{k} \xi_{i_{l} i_{k+1}}\right) \prod_{1 \leq a<b \leq k} \xi_{i_{a} i_{b}}\right]= \\
=\sum_{r=1}^{k+1}\left[\left(\prod_{\substack{l=1 \\
l \neq r}}^{k+1} \xi_{i_{l} i_{r}}\right) \sum_{S_{k}} \prod_{\substack{a \neq r \neq b \\
1 \leq a<b \leq k+1}} \xi_{i_{a} i_{b}}\right]=I_{k} \sum_{r=1}^{k+1} \prod_{\substack{l=1 \\
l \neq r}}^{k+1} \xi_{i_{l} i_{r}} .
\end{gathered}
$$

Therefore we should prove the identity

$$
[k+1]_{d}=\sum_{r=1}^{k+1} \prod_{\substack{l=1 \\ l \neq r}}^{k+1} \xi_{i_{l} i_{r}}=\sum_{r=1}^{k+1} \prod_{\substack{l=1 \\ l \neq r}}^{k+1}\left(d-b_{i_{l} i_{r}}\right) .
$$

This identity follows from the relation

$$
\sum_{r=1}^{m} \prod_{\substack{l=1 \\ l \neq m}}^{m} b_{i_{l} i_{r}}=\lambda^{m-1} \quad(m \leq k+1),
$$

which can be obtained by induction. Indeed, from Eqs.(3.7), (3.10) we have for $m=2,3$

$$
b_{i_{1} i_{2}}+b_{i_{2} i_{1}}=\lambda, \quad b_{i_{2} i_{1}} b_{i_{3} i_{1}}+b_{i_{1} i_{2}} b_{i_{3} i_{2}}+b_{i_{1} i_{3}} b_{i_{2} i_{3}}=\lambda^{2} .
$$

Then we deduce

$$
\begin{gathered}
\prod_{l=2}^{m} b_{i_{l} i_{1}}=b_{i_{2} i_{1}}\left(\lambda^{m-2}-\sum_{r=3}^{m}\left(\lambda-b_{i_{r} i_{1}}\right) \prod_{\substack{l=3 \\
l \neq r}} b_{i_{l} i_{r}}\right)= \\
=b_{i_{2} i_{1}} \sum_{r=3}^{m} b_{i_{r} i_{1}} \prod_{\substack{l=3 \\
l \neq r}} b_{i_{l} i_{r}}=\sum_{r=3}^{m}\left(\lambda^{2}-b_{i_{1} i_{2}} b_{i_{r} i_{2}}-b_{i_{1} i_{r}} b_{i_{2} i_{r}}\right) \prod_{\substack{l=3 \\
l \neq r}} b_{i_{l} i_{r}}= \\
=\lambda^{m-1}-\sum_{r=2}^{m} \prod_{\substack{l=1 \\
l \neq r}} b_{i_{l} i_{r}},
\end{gathered}
$$

which proves (A.4). Expanding the right hand side of (A.3) in power series of $d$ and taking into account (A.4) we verify the relations (A.3) and, thus, complete the proof.

One can reformulate the statement of Proposition A in more concise form (only in terms of elements $\xi_{i j}$ )

Proposition B Let $\xi_{i j}$ satisfy

$$
\xi_{i j}+\xi_{j i}=[2]=\xi_{i j} \xi_{j k} \xi_{k i}+\xi_{i k} \xi_{k j} \xi_{j i}(i \neq j \neq k \neq i) .
$$

We rewrite these conditions as

$$
\sum_{r=1}^{k} \prod_{\substack{l=1 \\ l \neq r}}^{k} \xi_{i_{l} i_{r}}=[k] \text { for } k=2,3 .
$$


Then, equation A.5) is also valid for $4 \leq k \leq n$, and the following identity holds:

$$
I_{k} \equiv \sum_{S_{k}} \prod_{1 \leq a<b \leq k} \xi_{i_{a} i_{b}}=[k] !,
$$

where $S_{k}$ denotes all permutations of the indices $\left(i_{1}, \ldots, i_{k}\right)$ and $i_{a} \neq i_{b}$ for $a \neq b$.

Proof The proof is similar to that of Proposition A.

Remark There are many other interesting relations among the elements $b_{i j}$ (3.7), (3.10) (as well as among $\xi_{i j}$ ). For example, one can easily deduce the identity

$$
b_{i_{1} i_{2}} b_{i_{2} i_{3}} \ldots b_{i_{k-1} i_{k}} b_{i_{k} i_{1}}=(-1)^{k} b_{i_{1} i_{k}} b_{i_{k} i_{k-1}} \ldots b_{i_{3} i_{2}} b_{i_{2} i_{1}},
$$

which generalizes (3.10) and follows from the relation

$$
-\frac{b_{j i}(p)}{b_{i j}(p)} q^{2 p_{i j}}=\pi_{i j} .
$$

Note that we consider $\pi_{i j}$ as constants which are independent of $p_{i}$.

\section{References}

[1] L.D. Faddeev, N. Yu. Reshetikhin and L.A. Takhtajan, Algebra i Analiz 1:1 (1989) 178 (English translation: Leningrad Math. J. 1 (1990) 193).

[2] J.-L. Gervais and A. Neveu, Nucl. Phys. B238 (1984) 125.

[3] E. Sklyanin, Algebra i Analiz 6 (1994) 227 (English translation: St. Petersburg Math. J. 6:2 (1995) 397-406), hep-th/9308060.

[4] A.G. Bytsko and L.D. Faddeev, J. Math. Phys. 37 (1996) 6324, q-alg/9508022.

[5] A.Yu. Alekseev and L.D. Faddeev, Commun. Math .Phys. 141 (1991) $413-422$.

[6] L.D. Faddeev, Commun. Math. Phys. 132 (1990) 131.

[7] A.N. Kirillov and N.Yu. Reshetikhin, Representations of the algebra $U_{q}(s l(2)), q$-orthogonal polynomials and invariants of links, in: Infinite Dimensional Lie Algebras and Groups, Proc. 1988 CIRM Conference, Ed. V. Kac (World Scientific, Singapore 1989), pp. 285-339.

[8] O. Babelon, D. Bernard and E. Billey, Phys. Lett. B375 (1996) 89-97, q-alg/9511019;

O. Babelon, Commun. Math. Phys. 139 (1991) 619-643.

[9] E. Witten, Commun. Math. Phys. 92 (1984) 455.

[10] A. Alekseev and S. Shatashvili, Commun. Math. Phys. 133 (1990) 353. 
[11] L.D. Faddeev, Gargèse lectures 1991, in: New Symmetry Principles in Quantum Field Theory, Ed. J. Fröhlich et al., Plenum Press, NY (1992), pp. 159-175.

[12] J.-M. Maillet, Phys. Lett. B162 (1985) 137.

[13] A.Yu. Alekseev and I.T. Todorov, Nucl.Phys. B421 (1994) 413.

[14] A.G. Bytsko, Fusion of q-tensor operators: quasi-Hopf-algebraic point of view, q-alg/9609007.

[15] A.G. Bytsko and V. Schomerus, Vertex operators - from a toy model to lattice algebras, q-alg/9611010.

[16] G.Felder, Elliptic quantum groups, in: Proceedings of the ICMP, Paris 1994. pp. 211-218. Ed. D. Iagolnitzer. International Press, Boston, 1995, and hep-th/9412207; Conformal field theory and integrable systems associated with elliptic curves, in: Proceedings of the ICM, Zurich, 1994, and hep-th/9407154.

[17] J.Avan, O.Babelon and E.Billey, Comm. Math. Phys. 178 (1996) 281; G. Arutyunov, L. Chehov and S. Frolov, R-matrix quantization of the elliptic Ruijsenaars-Schneider model, q-alg/9612032;

G. Felder and A.Varchenko, Elliptic quantum groups and Ruijsenaars models, q-alg/9704005.

[18] A.P. Isaev, J. Phys. A: Math. Gen. 29 (1996) 6903.

[19] P. Etingof and A. Varchenko, Solutions of the quantum dynamical Yang-Baxter equation and dynamical quantum groups, q-alg/9708015.

[20] P. Furlan, L.K. Hadjiivanov and I.T. Todorov, Canonical approach to the quantum WZNW model, ICTP Trieste and ESI Vienna preprint IC/95/74, ESI 234 (1995).

[21] P.Furlan, L.K.Hadjiivanov and I.T. Todorov, Nucl. Phys. B474 (1996) 497, hep-th/9602101;

P.Furlan, L.K.Hadjiivanov and I.T. Todorov, Int. J. Mod. Phys. A12 (1997) 23, hep-th/9610202.

[22] M. Dubois-Violette and I.T. Todorov, Lett. Math. Phys. 42 (1997) 183, hep-th/9704069.

[23] K. Gawȩdzki, Commun. Math. Phys. 139 (1991) 201;

F. Falceto and K. Gawȩdzki, J. Geom. Phys. 11 (1993) 251.

[24] P. Furlan, L.K. Hadjiivanov, A.P. Isaev, P.N. Pyatov and I.T. Todorov (in preparation).

[25] D.I. Gurevich, Algebraic aspects of the quantum Yang-Baxter equation, Algebra i Analiz 2 (1990) 119-148 (English translation: Leningrad Math. J. 2 (1991) 801-828). 
[26] P. Martin, Potts Models and Related Problems in Statistical Mechanics, World Scientific, Singapore, 1991.

[27] V.G. Drinfeld, Quantum groups, in: Proceedings of the International Congress of Mathematicians, Berkeley 1986, Academic Press (1986), Vol.1, pp. 798-820.

[28] M. Jimbo, Lett.Math.Phys. 11 (1986) 247.

[29] V.G.Drinfeld, Algebra i Analiz 1:6 (1989) 141 (English translation: Leningrad Math. J. 1 (1990) 1419);

N.Yu.Reshetikhin, Lett. Math. Phys., 20 (1990) 331.

[30] Yu.I. Manin, Quantum groups and noncommutative geometry, Université de Montréal preprint CRM-1561 (1989); Comm. Math. Phys. 122 (1989) 163-175.

[31] P.N. Pyatov and P.A. Saponov, J. Phys. A: Math. Gen. 28 (1995) 4415-4421;

D.I. Gurevich, P.N. Pyatov and P.A. Saponov, Lett. in Math. Phys. 41 (1997) 255-264;

A.P. Isaev, O.V. Ogievetsky, P.N. Pyatov and P.A. Saponov, Characteristic polynomials and Newton identities for quantum matrices, Preprint CPT-97/P3471 (1997).

[32] G.Felder and A.Varchenko, Comm. Math. Phys. 181 (1996) 741.

[33] E. Cremmer and J.-L. Gervais, Comm. Math. Phys. 134 (1990) 619;

A. Bilal and J.-L. Gervais, Nucl. Phys. B318 (1989) 579.

[34] P.P. Kulish and R. Sasaki, Prog.Theor.Phys. 89 (1993) 741.

[35] O. Babelon, Phys. Lett. B215 (1988) 523;

B. Blok, Phys. Lett. B233 (1989) 359.

[36] M. Chu, P. Goddard, I. Halliday, D. Olive and A. Schwimmer, Phys. Lett. B266 (1991) 71;

M. Chu and P. Goddard, Nucl. Phys. 445 (1995) 145. 